\title{
Ambiances
}

anbiances Environnement sensible, architecture et espace urbain Varia | 2016

\section{Le silence de la vulnérabilité en institution gériatrique}

The silence of vulnerability in geriatric institution

\section{Martine Leroux}

\section{OpenEdition}

Journals

Édition électronique

URL : http://journals.openedition.org/ambiances/696

DOI : 10.4000/ambiances.696

ISSN : 2266-839X

\section{Éditeur :}

Direction Générale des Patrimoines - DAPA - MCC, UMR 1563 - Ambiances Architectures Urbanités $(\mathrm{AAU})$

Référence électronique

Martine Leroux, "Le silence de la vulnérabilité en institution gériatrique », Ambiances [En ligne], Varia, mis en ligne le 27 avril 2016, consulté le 23 avril 2019. URL : http://journals.openedition.org/ ambiances/696 ; DOI : 10.4000/ambiances.696

Ce document a été généré automatiquement le 23 avril 2019

\section{cc) (†)}

Ambiances is licensed under a Creative Commons Attribution-NonCommercial-NoDerivatives 4.0 International License. 


\title{
Le silence de la vulnérabilité en institution gériatrique
}

The silence of vulnerability in geriatric institution

\author{
Martine Leroux
}

1 La vulnérabilité des vieilles personnes sonne comme une évidence ! On devient vulnérable avec l'âge, même si toutes les études et recherches sur le vieillissement montrent la diversité de ce processus et critiquent les normes et représentations sociales qui décident qu'une personne est vieille ou non. Reprenant la définition courante du terme, Jacques Richard, psychogériatre, écrit: "Est vulnérable ce qui est susceptible d'être attaqué, blessé ou endommagé. Lorsqu'elle qualifie l'être humain, la vulnérabilité signe sa fragilité » (Richard, 2004, p. 118). Ces deux notions, vulnérabilité et fragilité ${ }^{1}$, ont valeur de synonymes; c'est la seconde qui aujourd'hui fédère les acteurs du champ gériatrique. Nous préférons toutefois la notion de vulnérabilité qui dit, plus que la fragilité, la relation avec l'environnement immédiat, habituel. Parler de la vulnérabilité du sujet âgé renvoie donc à la fragilité observable des points de vue médical, social, psychique, etc., et indique un rapport au milieu de vie qui s'enracine dans la perte. La vulnérabilité naît dans la mise à l'épreuve, exprime la crainte de l'action et se traduit par un sentiment d'impuissance. Elle risque de s'installer durablement, d'empêcher l'adaptation à l'environnement et de bloquer toute velléité de résilience. L'entrée en institution peut-elle offrir un mieux-être, rassurer en palliant les difficultés? Le changement de milieu de vie ne risque-t-il pas au contraire d'aggraver et de renforcer la vulnérabilité objective et vécue, la transformant en dépendance? Nous pouvons alors nous demander: que fait l'institution de la vulnérabilité?

2 En quoi est-il pertinent d'appréhender cette notion sous l'angle du silence? Précisons notre perspective : s'intéresser au silence de la vulnérabilité, c'est orienter notre regard et notre réflexion ${ }^{2}$ sur la présence vulnérable qui se vivrait sur un mode silencieux. La notion de présence telle que nous l'entendons ici renvoie à Binswanger, psychiatre suisse, qui «cherche dans les dimensions fondamentales de l'acte d'exister les conditions de possibilité de l'être malade» (1971, préface, p. 7). La "présence», traduction du terme Dasein qu'il préconise, est « présence à », et elle s'ouvre toujours sur le monde selon une 
tonalité qui manifeste « l'indissociable unité du vécu et du monde » (2002, p. 42). Ce que l'homme vit et exprime, c'est toujours la manière dont il est disposé, à tel moment, dans telle situation. Notre intérêt pour le vécu de la vulnérabilité nous invite à considérer l'humeur des présences qui dit aussi la couleur des ambiances gériatriques, car l'ambiance, comme le rappelle Jean-François Augoyard s'interrogeant sur la construction des atmosphères quotidiennes du point de vue culturel, « consiste en la manifestation des "se montrer" par lesquels nous existons" (Augoyard, 2007-2008, p.60). Nous envisagerons d'un point de vue phénoménologique les deux formes du silence de la vulnérabilité dont deux expressions rendent compte : "rester sans voix " et "faire silence ». Avant de nous demander si l'institution ne met pas au silence les présences vulnérables, nous questionnerons la valeur heuristique de l'ambiance.

\section{Se sentir vulnérable - rupture et stratégie d'évitement}

\section{Rester sans voix - rupture de l'évidence vitale et identitaire}

3 «Les capacités d'adaptation ne cessent de s'abraser et de rendre le sujet âgé de plus en plus vulnérable» (Bouisson, 2005, p.51). Le changement progressif du rapport qu'entretiennent les personnes âgées avec leur milieu de vie est ici énoncé dans son objectivité, sous la forme du constat d'un inéluctable déclin. Mais le " sujet âgé » se sent-il devenir vulnérable ? La vulnérabilité, disions-nous, se vit sur un mode silencieux qui, du point de vue temporel, est d'abord la saisie de soi dans l'épreuve. Elle met en jeu la résistance du corps et de l'esprit qui se découvrent affaiblis dans le monde, impuissants à agir. La maladie est l'une de ces épreuves qui touchent directement la vitalité et ce que Claire Marin appelle l'« habitude d'être » (2004, p. 149). Cette philosophe qui s'interroge sur l'expérience de la maladie qu'elle a traversée, dit l'acuité de la vulnérabilité :

La vie reste discrète lorsque l'existence va de soi. Ainsi vécue sur le mode de l'évidence, elle n'a pas à être questionnée. Elle reste une entité abstraite, un chapitre de manuel. Elle n'est pas un problème, mais le postulat ininterrogé de toute présence. [...] Mais elle devient parfois surprise et déception, lorsque le corps se dérobe et déserte son poste de vigie. Le gardien du moi semble le trahir et l'abandonner à sa seule faiblesse. Cette défense désarmée, le sujet n'est plus que vulnérabilité, plaie ouverte. (Marin, 2008, p. 8)

Ici le silence de la vulnérabilité signifie au sens propre : rester sans voix dans l'actualité d'une rupture de l'évidence vitale qui se révèle en quelque sorte après une bataille dont l'être sort vaincu, remet en question l'identité du sujet et contrarie le rapport au monde de la présence. Rester sans voix, c'est interrompre le flux des paroles et des pensées, comme si la force que requiert la parole se dérobait. Deux questions se posent : la prise de conscience de soi comme être vulnérable met-elle toujours en jeu avec autant de violence l'assise existentielle, ou peut-il y avoir un apprentissage de la vulnérabilité ? Par ailleurs y a-t-il une spécificité - circonstances, motifs... - de ce saisissement avec l'avancée en âge ?

$5 \quad$ Les vieilles personnes peuvent certes connaître cette expérience de vulnérabilité causée par la maladie, mais beaucoup sont plutôt atteintes par des fragilités « acquises » au cours de la vie qui les conduisent au fil des jours à abandonner certaines pratiques, à adapter certains gestes, à amorcer le deuil d'une image d'elles-mêmes. «Le lien avec l'âge est réalisé très spontanément: il est vraisemblable que l'apprentissage de la vieillesse, en tant que limitation des performances corporelles, commence très tôt dans la vie des individus." (Clément, Mantovani \& Membrado, 1995, p.10) Ainsi les personnes 
s'habitueraient aux pertes progressives de leurs capacités et échapperaient à ce sentiment vif de vulnérabilité que nous venons d'évoquer. On peut penser que certaines positions profondes par rapport à la vie, au temps qui passe... adoucissent les prises de conscience qui surviennent au cours de la vie dans des moments de rupture et facilitent l'acceptation de soi diminué. À cet égard, les études sociologiques auprès des personnes de grand âge montrent que «les uns considèrent qu'ils deviennent vieux sans l'être encore, alors que d'autres reconnaissent que désormais ils sont vieux ", et ceux-là " ont le sentiment d'une rupture dans leur existence - qu'ils parviennent souvent à dater - et d'être devenus autres qu'ils étaient » (Caradec, 2012, p. 108).

N'est-ce pas la découverte de soi comme " être vieux » qui constitue, avec l'avancée en âge, la spécificité de la prise de conscience de soi comme être vulnérable? D'une part, si bien des événements peuvent rendre vulnérables au cours d'une vie, ils deviennent plus fréquents avec l'âge. D'autre part, le vieillissement, dont on pourrait penser qu'il ne fait que connoter une de ces ruptures, ne constitue-t-il pas un événement? Plusieurs facteurs interviennent. Du point de vue temporel, ce n'est pas juste le vertige de l'irréversibilité qui se manifeste avec plus d'acuité. C'est la visibilité soudaine de cette irréversibilité que le corps, le visage dévoilent. François Jullien évoque le paradoxe des "transformations silencieuses ", et « devant la photographie d'il y a vingt ans, c'est cette validité du "sujet" qui soudain défaille » (Jullien, 2009, p. 15). De nombreux romans évoquent cette atteinte à l'identité, qui passe entre autres par l'image de soi que le miroir bouscule. C'est la continuité et l'unité de la personne qui sont visées. "L'image de soi est fondée sur un principe d'unité de la personne, qui s'établit en une sorte de résumé biographique : elle dit (ou est censée dire) instantanément l'essentiel» (Kaufmann, 2005, p. 68). Comment alors intégrer, accepter cette nouvelle image de soi ? Ainsi, lorsque s'éprouve, à un âge avancé, la rupture de l'évidence vitale et identitaire dans la saisie de sa propre vulnérabilité, " rester sans voix » serait découverte de soi comme " être vieux », affaibli dans un monde désormais hostile. La vieillesse est essentialisée, et se sentir vieux vient fermer le monde et freiner le désir de résilience. L'entrée en institution, qui fait suite le plus souvent à des décisions prises par la famille, constitue un de ces événements capables de provoquer un ébranlement profond de l'être. Mais la saisie de soi comme être vulnérable et vieux, parce que vieux, intervient après coup, après la brutalité de l'affect ; c'est l'événement appréhendé dans le cours de sa propre histoire qui peut produire le sentiment d'une catastrophe.

7 Le sentiment de vulnérabilité trouve-t-il l'occasion de se réactualiser avec la même intensité? En situation, nous avons affaire à ce sentiment à la fois enraciné dans l'expérience affectante et engagé dans le présent de l'action: la présence vulnérable éprouve à ce moment-là de la peur, elle reste, là aussi, sans voix. En institution, dans les espaces collectifs, des scènes que nous avons pu observer manifestent cette actualisation: ainsi, lorsqu'au milieu de l'après-midi, dans l'ambiance encore tranquille de la fin de sieste, le kinésithérapeute sollicite une vieille dame légèrement assoupie dans le salon pour la séance de marche prévue, on décèle une expression apeurée sur le visage de celleci. Elle avancera tel un automate, le regard fixe, et n'entendra pas les encouragements que lui prodigue le kinésithérapeute. Car la voix et les propos tenus, qui relèvent du registre de la volonté, restent extérieurs à la peur. Le kinésithérapeute est dans son rôle, mais manque la relation avec la vieille dame qui retrouve intactes les difficultés qu'elle redoutait. Nous comprenons que le devenir du sentiment de vulnérabilité se trouve du côté de l'évitement. 
8 Nous retiendrons que le « rester sans voix » de la vulnérabilité, et ce quels qu'en soient les « causes » (physique, sociale, cognitive, environnementale, etc.) et les embrayeurs, se manifeste selon la temporalité de l'événement défini comme " ... ce à quoi on ne s'attend pas, ce qui sur-vient et vient ainsi sur nous par surprise, ce qui nous "tombe dessus", l'accident au sens propre » (Dastur, 2004, p. 165). La vulnérabilité perçue avec acuité se vit sur le mode de l'effondrement et de l'éloignement radical d'avec le monde d'avant connu et vivant. Comme dit plus haut, l'expérience du vieillissement n'est pas univoque; déni, accablement ou acceptation de ce que les personnes âgées considèrent comme naturel, ou encore désir d'agir, traduisent les multiples manières de réagir aux signes de la sénescence et de la vulnérabilité ressentie. Mais quel est le devenir de cette tonalité profonde de la vulnérabilité découverte brutalement sur le mode de l'affect? Une fois éprouvée, s'installe-t-elle dans la durée?

\section{Faire silence - adoption d'une stratégie défensive}

On peut se demander si le silence comme mode d'apparaître en maison de retraite est celui de la vulnérabilité et s'il ne s'affirme pas comme une stratégie défensive. Jean Bouisson, psychologue clinicien, s'interroge sur « la vulnérabilité perçue » des personnes âgées face à une situation de plus grande vulnérabilité (Bouisson, 2005 et 2007). En l'absence d'études sur cette question, il s'intéresse aux ouvrages consacrés aux situations extrêmes et note :

L'émergence du sentiment de vulnérabilité intervient dans un contexte particulier de pertes des repères les plus familiers, pertes pouvant se situer tout autant dans un environnement externe (décès des proches, perte des rôles sociaux) qu'interne (altération de l'état de santé, atteintes organiques, déficits cognitifs, etc.) et se traduisant généralement par un ébranlement identitaire plus ou moins grave et une chute de l'estime de soi. (Bouisson, 2007, p. 110)

Ce sentiment de vulnérabilité, lorsqu'il persiste, devient mode de vie. La peur des situations à risques se fait permanente et contamine tous les aspects de la vie quotidienne. Quelles stratégies adoptent alors ces personnes vulnérables qui développent des troubles anxieux, parmi lesquels la peur des relations sociales? Pour faire taire ce sentiment, elles recourent à des routines visant si possible à éviter les risques; quatre procédés typiques sont repérés : régression à des besoins infantiles, réduction du champ de la pensée et des activités, mise en place d'un ordre fixe et défense d'un territoire intime (Bouisson, 2007, p. 111-112). L'adoption de ces routines chez les personnes âgées conduit à une rigidité ne supportant aucun événement perturbateur dans l'ordre quotidien. Bouisson prend l'exemple, bien connu des visiteurs en institution gériatrique, de personnes " "rivées" pendant des heures sur "leur" fauteuil, le plus souvent inoccupées... » (Ibid., p. 115) ; elles occupent toujours la même place et réduisent leur espace de vie entendu aussi bien au sens géométrique que psychique. Bouisson distingue toutefois ce qu'il appelle la "routinisation processus", que nous venons d'entrevoir, de la "routinisation stratégie »; même si la marge entre les deux est très étroite, cette dernière peut opérer comme ressources personnelles d'adaptation.

Qu'expriment ces visages, pour beaucoup silencieux, que l'on découvre lorsqu'on entre dans une maison de retraite? Le silence peut être celui du repos plutôt apaisé en début d'après-midi, mais il peut être aussi celui de la retenue, voire du repli teinté de mécontentement. Les vieilles personnes dans les espaces collectifs ont tendance à se taire. Le silence du repli, plus qu'un mode sonore, apparaît comme une manière d'être qui 
engage tout le corps dans sa relation à l'environnement et à autrui. Se taire se traduit par une gestuelle prudente, plutôt de retrait. Dans cette situation collective qu'elles n'ont pas connue au cours de leur vie, les personnes ne cherchent-elles pas, par le silence, à préserver leur identité ? Nous avons rencontré des personnes qui se réfugient dans leur passé avec nostalgie. Elles aiment raconter à quelques soignants sur le mode de la confidence des bribes de leur vie, les événements marquants. D'autres personnes ne montrent pas cette expression un peu lointaine de la nostalgie, mais adoptent le visage de la politesse. D'autres encore ont une expression fermée et empêchent de la sorte tout échange. Il s'agit dans tous les cas de ne pas abdiquer leur identité.

On peut aussi se demander si bien des visages silencieux et des corps lourds qui sont réunis dans les salons des institutions gériatriques n'expriment pas juste la fatigue liée au grand âge. S'interrogeant sur «les sentiments corporels", Charbonneau note: "La fatigue altère la relation de déploiement que nous avons vis-à-vis du monde. En réduisant l'ampleur du monde déployé, elle accentue notre pesanteur - la pesanteur étant l'expression de l'anti-déploiement de notre être » (Charbonneau, 2003, p. 22). La fatigue ne devient-elle pas alors une manière d'être moins douloureuse que la vulnérabilité ressentie, au risque de se transformer en fatigue de vivre?

Il est encore un silence de la vulnérabilité à l'œuvre dans la construction du discours et dans la présentation de soi : celui qui cherche à masquer l'oubli d'un mot, d'une idée, d'un souvenir qui a laissé une trace mais trop éloigné dans la mémoire pour être raconté. Selon Jean Bouisson, on sait que " [...] de nombreux sujets ont une perception confuse d'une altération de leurs performances. Spontanément d'ailleurs, beaucoup cherchent à masquer leurs pertes à leur entourage, peut-être pour se tromper eux-mêmes par la même occasion. » (Bouisson, 2007, p. 119). L'inquiétude qui accompagne le déclin des performances, cognitives en particulier, accentue le sentiment de vulnérabilité. Nous pensons aux présences hésitantes que nous rencontrons dans les espaces collectifs de l'institution, aux regards parfois interrogateurs, ou qui craignent de recevoir la réprobation, aux expressions incertaines, comme tremblées...

En dépit d'une singularité que les visages expriment, les vieilles personnes laissent souvent entrevoir la difficulté d'être là, comme si elles étaient un peu perdues, et leur silence, défini d'abord comme non parole, manifeste une prudence vis-à-vis d'autrui et une réserve qui peuvent se figer ou s'ouvrir peu à peu. L'évitement par le silence ne risque-t-il pourtant pas de s'épuiser dans l'inefficacité et dans la fermeture sur le monde? Faire silence, n'est-ce pas alors vivre sur un mode mineur et museler la vitalité même réduite que chacune des présences porte encore en elle, n'est-ce pas empêcher l'émergence de tout désir, et par conséquent enlever tout sens à l'existence ? Richard, dont la définition ouvrait notre propos, questionne la possibilité d'un sursaut pour s'adapter aux nouvelles situations : « Lors du vieillissement, les conduites résilientes et de vulnérabilité peuvent comporter des oscillations selon le niveau d'adaptation exigé, individuellement ou collectivement, à un moment donné par les situations rencontrées, mais aussi selon la conscience que l'on en a ( (Richard, 2004, p. 121). La résilience des personnes âgées ne serait-elle pas du côté de l'acceptation active plutôt que de l'affrontement? 


\section{Recourir à l'ambiance - la tonalité des « présences »} de son monde ; ainsi Binswanger écrit à propos du cas Suzan Urban, patiente qui vit sous «la puissance de la terreur » dans une atmosphère menaçante: «la présence perçoit comme monde extérieur ce qu'elle est originairement» (Binswanger, 2002, p. 43), et c'est un monde ambiant malmené par son esprit tourmenté qu'elle rencontre, mais qui toutefois n'est pas complètement coupé, isolé du monde alentour - la psychose interroge d'ailleurs sur l'impossible accueil du monde et éclaire en ce sens les modes d'être qui y donnent accès. La spatialité, à laquelle Binswanger reconnaît un rôle primordial ${ }^{4}$ dans l'expression du sens de l'existence, permet de saisir ce rapport intrinsèque entre la dimension thymique et le monde environnant; celui-ci est appréhendé sous sa forme spatiale en tant qu'«espace orienté, géométrique et physique» toujours perçu «relativement à l'engagement psycho-moteur et corporel du Dasein» (Gros in Binswanger, 1998, p. 22). Et nous comprenons que cet engagement n'est pas sans effet sur l'ambiance et réciproquement. Commentant la conférence de Binswanger sur l'espace, Caroline Gros note: «La spatialité s'inscrit dans cette dimensionnalité de l'affection thymique dans laquelle les sentiments ne concernent plus seulement la subjectivité isolée, mais se propagent vers le monde en écho et reviennent du monde vers soi par une boucle de réverbération» (Ibid., p. 39). Comment saisir alors la dimension spatiothymique de la présence? Binswanger, à l'écoute des malades et de leurs rêves, s'est intéressé aux « directions de sens $»^{5}$ qui orientent la présence. Les vieilles personnes que nous avons rencontrées ne recourent pas à des métaphores ${ }^{6}$ pour exprimer ce que la vulnérabilité a provoqué en elles. Mais en revanche, celle-ci entraîne le corps, le freine, l'immobilise..., elle se fait geste selon un certain rythme, selon une temporalité vécue associée à la spatialité.

Reprenant les distinctions que propose Minkowski pour analyser le temps vécu, nous pouvons nous demander si le "principe de déploiement ", qui désigne «ce passage, dans la vie, de la succession à la continuité» (Minkowski, 1968, p. 247), n'est pas bloqué, imposant une conscience du temps fragmentée, réduite à des « maintenant » sans passage au " présent », ce dernier étant défini comme « un maintenant qui s'est déployé » (p. 32). Il est intéressant également d'envisager les deux principes opposés qui constituent pour Minkowski le point de départ de ses recherches sur la phénoménologie du temps et sur l'aspect temporel des troubles mentaux: au temps mesuré de la vie 
quotidienne selon le «principe de juxtaposition » s'oppose le temps des vacances selon le "principe de pénétration $»^{7}$ par lequel «nous nous confondons entièrement avec la vie ambiante ». Et les deux principes ne se limitent pas au temps, ils concernent « aussi bien la contexture de l'ambiance que nos rapports avec l'ambiance » (Minkowski, 1932, p. 233).

Avant de poursuivre, il est important d'introduire la dimension de l'altérité, qui constitue également un des facteurs clés de l'ambiance. Elle n'est pas séparée de la dimension spatiale. «L'entente spatiale de l'être-au-monde ne peut se comprendre qu'en tant que thymiquement disposée par l'altérité. L'autre, d'une façon qui n'est jamais neutre, médiatise notre rapport à l'espace ", écrit Caroline Gros (2009, p. 238). L'autre, c'est bien sûr celui que nous nous représentons, avec lequel nous cherchons à maintenir une plus ou moins grande distance et qui va susciter un sourire, une parole, ou au contraire un détournement de tête, un rictus... L'engagement du corps, dans un espace médié par autrui et dans un présent plus ou moins ouvert sur l'avenir, indique le rapport à l'ambiance et indique aussi ce que l'ambiance porte en elle de stimulant ou de contraignant. La notion de «sentiment d'aisance » que Minkowski adopte à propos de l'espace public (1968, p. 374) nous semble pertinente ici pour rendre compte de la manière dont les présences se déplacent et se positionnent dans les espaces collectifs de l'institution.

Le silence de la vulnérabilité qui accompagne nombre des actions des personnes âgées se charge de bien des significations, elles-mêmes conjuguées dans une humeur qui traduit la plus ou moins grande aisance d'être là. Dans les ambiances calmes ou animées selon le moment de la journée, l'attention portée à ce silence des présences peut rendre audible la vulnérabilité ; nous pouvons nous interroger aussi bien sur le bruit des préoccupations derrière le silence que sur le silence de la vulnérabilité derrière le bruit des propos répétitifs et le fond sonore environnant. Si nous reprenons les termes de Binswanger, ne pouvons-nous dire que la spatialité de la présence est désormais limitée au monde obligé de l'institution et recroquevillée en quelque sorte dans une attitude de protection? En outre cette présence ne vit-elle pas selon une «structure temporelle» incapable d'accepter le nouveau (Binswanger, 2002, p. 83) ? Nous pouvons nous demander en quoi ces positions ont à voir avec les ambiances de l'institution. En dépit d'une évolution vers plus d'individualisation, qu'est-ce qui pourtant résiste et représente toujours un risque qui relèverait de l'institution «totalitaire $»^{8}$ ? Nous ne perdrons pas de vue cette question reconnaissant que l'institution enferme, limite, interrompt la continuité vécue et exige une adaptation au collectif. Quels sont donc les facteurs propres aux ambiances de l'institution qui en constituent le fond ${ }^{9}$ et le potentiel toujours actualisés dans les différentes situations d'ambiance? Quels sont ces facteurs dans les trois dimensions qui se conjuguent dans le silence vécu par les vieilles personnes: spatialité, temporalité et altérité ?

\section{Mettre au silence - Que font de la vulnérabilité les ambiances de l'institution?}

Quelle est la marge de manœuvre dont les vieilles personnes disposent entre «leurs ressources intimes » et les « ressources extérieures » ? L'institution ne risque-t-elle pas de les «mettre au silence » et de réduire leur faible potentiel de résilience? 


\section{Une spatialité limitée et familière}

21 La question de la spatialité nous invite à considérer tout d'abord le rapport dedans/ dehors que le résident va percevoir une fois entré dans la maison de retraite. «Les expressions "être enfermé" ou "être dedans" ne prennent toute leur signification pour le reclus que par rapport à celles de "sortir" ou d'"aller dehors" ", écrit Goffman dans Asiles (1968, p. 56). Du point de vue spatial, qu'induit le terme «reclus » pour désigner les résidents? Il est indéniable que certains, en particulier ceux présentant des pathologies mentales, éprouvent ce sentiment. "Pourquoi on peut pas sortir, on nous tient... ", répète inlassablement cette femme d'une unité protégée dans un EHPAD à la recherche constante d'une brèche dans l'environnement. Dans ce type d'établissement, les résidents qui en ont encore la capacité apprécient de sortir accompagnés, mais ce qui est vécu comme une bouffée d'oxygène fatigue, et ils retrouvent avec ambivalence l'institution. Ils sont à nouveau dans ce monde fermé et protecteur. Ils renoncent au sens propre au dehors auquel ils ne peuvent plus accéder de manière autonome, mais dont le sens symbolique reste vif : ils doivent abandonner leur vie d'avant, familiale et sociale; en ce sens, regarder s'éloigner le parent venu les visiter, c'est éprouver la coupure entre dedans et dehors.

L'architecture la plus attentive peut-elle éviter ce sentiment d'enfermement qui, d'ailleurs, n'est pas omniprésent mais se rappelle à la conscience à certains moments de la journée, dans certaines circonstances? L'implantation des bâtiments, les extensions, les ouvertures peuvent maintenir un lien avec l'extérieur. Ainsi, l'aménagement du passage entre dehors et dedans peut atténuer le sentiment d'entrer dans une "résidence» comme posée là qui ne pourrait que conforter l'idée d'hétérotopie

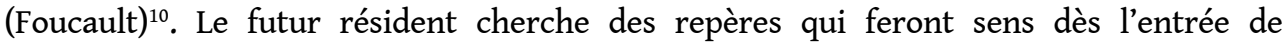
l'institution; dans les maisons de retraite situées en ville, le passage d'un sas entre deux portes d'entrée coulissantes ou une porte d'ascenseur qui se referme marquent trop brutalement la rupture avec l'extérieur. La « rue intérieure » de l'hôpital Bretonneau ou le hall d'un établissement partagé avec les habitants (Guislain, 2012, p. 60-63) ${ }^{11}$ peuventils faire transition? Par ailleurs, les extensions que représentent les jardins offrent comme une échappée aussi bien aux résidents qu'aux soignants et aux visiteurs - un jardin coincé dans un cœur d'ilot n'a d'ailleurs pas le même attrait que celui dont la grille donne sur l'espace public. Enfin, l'ouverture des baies vitrées, la vue sur le paysage alentour rattachent le résident à la ville ou à la campagne. Notons que cette ouverture sur l'extérieur ainsi que la couleur du temps si importante pour l'ambiance sont perçues dans les espaces collectifs plutôt que dans la chambre car les résidents y sont en relation, même sur le mode du silence, principalement avec les personnels; personnels dont la présence ou la proximité soutient en quelque sorte leur regard sur le monde. À cet égard, notons que les espaces fréquentés sont ceux qui, toujours, permettent ce lien. Nous avons vu des salons spacieux, conçus dans un souci d'offrande spatiale, désertés car trop éloignés des lieux de vie.

Dans ce monde fermé de l'institution, le reclus « s'approprie le lieu qu'il occupe, organise l'opposition du privé et du public, de l'intime (des dessous) et de l'exposé (du dessus). [...] Le reclus gère des territoires et des visibilités ", écrit Isaac Joseph, évoquant les figures différentes du reclus, dont celle du « résidant» (Joseph, 2001, p. 85). Ce sont donc les territoires possibles de l'institution elle-même qui mobilisent l'appropriation spatiale. 
Ainsi en va-t-il de la routinisation, qui commence par la place que l'on va prendre dans l'un des " points de densité » (Eynard \& Salon, 2006, p. 44) - hall de la maison de retraite, salon de son unité... Comment investir l'espace privatif de la chambre qui donne sur les espaces collectifs, menaçant de la sorte l'intimité, et qui se transforme en espace de soins à certains moments? Mais la localisation de la chambre peut déjà compromettre cet investissement - ainsi une dame se sent "délaissée » au bout du couloir. Comment s'approprier des espaces collectifs dont «l'emballage flatteur peut être plus ou moins hôtelier, hospitalier ou domestique» (p. 27)? Aujourd'hui le modèle domestique se développe "en consolidant l'espace privé de la chambre» et "en introduisant dans l'espace collectif de l'unité le simulacre des autres pièces du logement: séjour, salle à manger ou coin repas» (p. 134). Nous avons toutefois pu observer des résidents qui parviennent à créer leur " espace propre $»^{12}$ dans différentes unités, protégées ou non ; celui-ci s'effectue au cours de déplacements familiers selon une gradation privé-collectif et met en évidence la spatialité de l'habitude, qui « n'est pas inscrite à l'intérieur du corps biologique mais dans les lignes de ses mouvements et déplacements, dans les gestes " (Kaufmann, 2005, p. 85). Cet espace propre donne-t-il pour autant le sentiment du chezsoi ? Sans doute si nous considérons la possible émergence de ce sentiment dans des lieux et à des moments différents ; la définition du chez-soi en maison de retraite que propose Isabelle Mallon s'appuie sur cette capacité d'investissement liée à une personnalité: "C'est un lieu investi subjectivement par la personne, qui lui permet de se reconnaître et qui garantit son identité » (Mallon, 2004, p. 22). Mais créer dans les espaces privés et partagés de la maison de retraite son chez-soi suppose d'accepter de se trouver là en sachant qu'il s'agit le plus souvent du dernier lieu de vie.

\section{Une temporalité routinière et menacée}

La vie en institution se déroule selon une temporalité monotone. On pourrait parler d'un rituel des fonctions qui se substitue à toute autre activité et conforte, voire engendre, la routinisation dont nous parlions plus haut. L'adaptation un peu mécanique au rythme de l'institution qui est, dans le meilleur des cas, la voie de la familiarisation, confère aux habitudes acquises dans ce lieu un statut de routines. Se référant à Ricœur, Claire Marin rappelle deux versants de l'habitude « entre l'habitude comme puissance de facilitation ou comme chute dans l'automatisme»; le deuxième l'emporterait, privilégiant la répétition dénuée de sens du geste (Marin, 2004, p. 152). «La rupture des rôles sociaux » ${ }^{13}$ (Gaucher, 1982a) qui caractérise le vieillissement se radicalise ici et favorise la perte des repères spatiaux et temporels. "Le rôle de résident est marqué par l'indétermination ", écrit Isabelle Mallon (2007, p. 49), car l'institution se donne pour mission l'« accompagnement » et prend en charge tous les besoins de la personne. L'entrée en institution a des répercussions sur la mémoire et la temporalité vécue, selon Jacques Gaucher qui fait l'hypothèse qu'ainsi « la difficulté de se souvenir des événements récents de même que les perturbations de l'attention seraient la conséquence d'une rupture temporelle de soi et des rôles sociaux qui y sont associés... » (Gaucher, 1982b, p. 85). La confusion temporelle de nombre de personnes âgées risque d'apparaître ou de se confirmer en institution. Elles manifestent alors une désorganisation du temps vécu et notamment de l'axe chronologique, qui « repose sur la mémoire, source et organisation du passé, et sur l'idée de projet, organisateur de l'avenir " (1982a, p. 50). Le futur des vieilles personnes, limité de fait, ne nourrit pas le présent, qui va puiser dans le passé mais, demande Jacques Gaucher : «Comment concevoir que le souvenir puisse s'organiser 
ou persister sans le secours organisationnel du présent ? Si la notion de présent disparaît, le souvenir disparait » (p. 51). On peut s'interroger à juste titre sur le "projet de vie", doublé d'un « projet de soins », qui doit accompagner l'entrée en institution.

En référence aux analyses de Minkowski, nous pouvons dire que le présent vécu s'ancre désormais davantage dans le "principe de juxtaposition" que dans celui de "pénétration ». Du point de vue existentiel, que devient alors le présent? Les personnes âgées risquent-elles d'entrer dans ce temps long et angoissant de l'ennui, qui est une "altération du "vécu de valoir", de la valuation des choses... » (Charbonneau, 2003b, p.30)? Quiconque fréquente régulièrement ou ponctuellement les institutions gériatriques peut se poser cette question en voyant les vieilles personnes assises tout l'après-midi, inactives et sans velléité de bouger ou de participer aux activités proposées. Elles disent souvent : «j'attends... », et précisent en général l'objet de leur attente - le goûter, le soignant, etc. L'attente dans ce cas s'inscrit dans du temps mesurable et échappe ainsi à l'anxiété qui est inhérente à « l'attente primitive » définie comme « une suspension de l'activité qui, elle, est la vie même » (Minkowski, 1995, p. 80). L'attente devient alors activité; trois ethnologues, immergés six mois en EHPAD, constatent : "À rebours de ce que l'on pourrait penser a priori, la passivité associée habituellement à l'attente n'est en aucun cas synonyme de vacuité. Ce temps d'attente est directement en rapport avec l'action» (Jaujou, Minnaërt \& Riot, 2006, tome 1, p. 187). Ainsi la vie se manifeste encore dans son déploiement réduit, et la contradiction principielle entre « deux chemins qui bifurquent, l'un inépuisable et l'autre qui va vers le bas » (Minkowski, 1968, p. 130-131) ${ }^{14}$ s'atténue peut-être, mais au détriment du présent qui pourtant toujours revendique; car, comme l'écrit Ricœur à la fin de sa vie: "Ce qui occupe la capacité de pensée encore préservée, ce n'est pas le souci de ce qu'il y a après la mort, mais la mobilisation des ressources les plus profondes de la vie à s'affirmer encore.» (2007, p. 41).

Dans le temps monotone de l'institution, certaines manifestations se vivent pourtant selon le " principe de la pénétration » et parviennent à faire mémoire comme événement joyeux. Ainsi, plusieurs personnes évoquent la chorale venue à l'occasion de Noël ou encore l'ambiance festive de carnaval qui rassemblent ponctuellement résidents, soignants, visiteurs. Des espaces fréquentés régulièrement favorisent alors dans ce contexte ce que Maldiney appelle le "transpassible $»^{15}$ qui indique la réceptivité à l'événement. Jean Oury, psychiatre, définit cette notion comme le contraire de "l'impassible», de "ce qui ne bouge pas» (Oury \& Depussé, 2003, p. 68). Or, dit-il, s'interrogeant sur l'institution psychiatrique, «pour qu'il se passe quelque chose, il faut travailler la disposition des choses, l'architectonie.» (p. 68); il faut parvenir à une « distinctivité » entre des lieux et il précise, se référant alors à l'école, que les couleurs différentes des salles de classe ne suffisent pas à la créer " parce qu'il restera l'odeur de l'école» (p. 69). Dans quel lieu de l'institution gériatrique, autre que celui de l'unité de vie, de la «maisonnée » - conçue en général pour une quinzaine de personnes - est-il possible de «bouger » l'ambiance et de provoquer la rencontre ? Le plus souvent, il s'agit d'espaces familiers susceptibles de transformation comme la salle à manger ou le salon d'un établissement qui par leur distinctivité d'un jour engendrent le plaisir d'être là et, en retour, s'imprègnent des ambiances partagées. 


\section{Une altérité en miroir et énigmatique}

27 On a vu que les personnes âgées craignent de perdre leur identité et se trouvent confrontées au miroir que leur renvoient les autres; elles découvrent aussi ce que Goffman appelle les "inconvenances situationnelles $»^{16}$ qui ont trait à l'intimité, aux propos confus chez certains, à la «folie». Certaines d'entre elles parviennent à développer et entretenir des relations privilégiées avec une ou deux autres personnes, mais en EPHAD nous avons souvent observé le manque d'échanges spontanés entre les résidents. Au cours des jeux de société, nous entendons parfois des propos peu amènes. Les ambiances manifestent d'ailleurs l'absence de lien ; si la tranquillité du début d'aprèsmidi correspond à une pause bienvenue, elle apparaît ensuite bien fragile dans nombre d'unités des maisons de retraite, comme si le calme rencontré ne faisait pas enveloppe mais se composait des préoccupations silencieuses. Dans les espaces collectifs, les présences assoupies ou éveillées, certaines à l'affût du moindre geste ou événement qui fera diversion, d'autres perdues dans leurs pensées, semblent réunies là comme accidentellement. Est-ce un sentiment de solitude que la situation collective contredit qui alourdit l'ambiance et rend perceptible l'hétérogénéité du non-dit? Plusieurs points méritent d'être signalés qui manifestent la riche palette des ambiances et la création possible du lien. Ainsi il suffit d'un jour d'été pour que, dans un salon, à l'abri du soleil, les présences réunies communiquent dans le calme du fond sonore du téléviseur, comme si la pénombre permettait l'individuation et la proximité. En revanche, dans le salon ensoleillé, la lumière semble isoler les présences et le silence devient vacuité. Les choses elles-mêmes semblent atteintes de mutisme et le monde risque de se néantiser. Par ailleurs il convient de nuancer ce que la non parole de nombre de résidents peut induire : en effet l'émergence inattendue d'une remarque à l'égard d'autrui manifeste la discrétion plutôt que l'absence des personnes rassemblées - nous pensons à une résidente qui s'adresse de loin à cette femme dont nous parlions plus haut, marchant avec peur et raideur sous l'œil du kinésithérapeute : « Vous avez fait des progrès, Mme X. »

C'est vers les professionnels que les vieilles personnes se tournent pour obtenir reconnaissance et gestes d'intérêt à leur égard. La logique de gestion ne constitue-t-elle pas un obstacle à l'accompagnement des résidents, aux pratiques du care ${ }^{17}$ ? Selon Iris Loffeier (2011), qui analyse les "tensions entre missions chez les personnels d'Établissements d'hébergement pour personnes âgées dépendantes » à partir de la grille d'analyse des cités (Boltanski, Thévenot, 1991), «tous les personnels s'inscrivent de la même manière dans la cité industrielle de l'efficacité »(p. 83). La temporalité à deux vitesses que nous observons confirme ce diagnostic: la rapidité des professionnels, soignants et administratifs, toujours dans l'action, croise la lenteur des vieilles personnes. Iris Loffeier souligne également les injonctions divergentes entre les catégories de personnels : "Alors que la cité domestique permet l'utilisation d'une autorité sur les petits, la cité marchande, dans laquelle s'inscrivent principalement les gestionnaires, interdit cette domination sur l'individu payeur » (p. 82). Selon elle, nous avons affaire ici à la "cité domestique " parce que "la vieillesse est vue comme un retour en enfance " (p. 82) ; elle pointe ici une représentation tenace du grand âge qui justifie à la fois attendrissement et propos familiers et autoritaires, notamment de la part des aidessoignants en contact permanent avec les personnes âgées. Cette représentation met en jeu l'inversion des rôles de la relation parents-enfants qui peut conduire, nous dit Ploton, psycho-gériatre, à une « sensation de toute puissance » (2001, p. 13). Mais, la vulnérabilité 
inscrit dans la relation entre résidents et soignants une asymétrie que l'on trouve au cœur de la relation de soin : "Il n'y a pas de soin sans une relation entre une faiblesse qui appelle de l'aide, mais qui peut devenir une soumission, et une capacité qui permet le dévouement mais qui peut devenir un pouvoir, et même un abus de pouvoir » (Worms, 2006, p. 142). C'est dans cette brèche que s'engouffre le risque de maltraitance que le grand âge et la nature des tâches à accomplir peuvent favoriser et c'est dans le geste du soin qu'une symétrie peut s'instaurer. Les séquences filmées par Nicolas Archimbaud et les analyses qu'il en fait - sa thèse (2013) s'intitule Vie quotidienne et soins des personnes âgées dépendantes en milieu hospitalier - montrent « la subtilité des interactions observées entre soignants et soignés » (p. 478) qui permet au "corps-objet » de devenir " corps-sujet » lors des « techniques de ré-éducation, ré-adaptation, ré-assurance ».

Nous avons vu que la vulnérabilité n'est pas seulement celle des corps que l'institution aime mettre en avant dans l'offre qu'elle propose, même si le soin des corps constitue une médiation vers les "présences». Le silence de la vulnérabilité qui a retenu notre attention est celui de l'épreuve et de l'évitement. Il n'est pas signification à comprendre, il exprime ce qui se joue dans la perte des repères, dans l'attente, dans l'angoisse de la mort. Ce silence de la vulnérabilité que les facteurs complexes du fond même de l'ambiance contribuent à mettre en place peut-il échapper à la rigidité qui le guette, nous demandions-nous. Comment susciter chez la personne âgée le désir de partager une situation, comment densifier et apaiser son présent alors que la vulnérabilité est passivité et que l'institution est là pour compenser l'incapacité ? Les routines et le repli évitent l'inquiétude qui, elle, se manifeste dans l'action à la recherche des indices qu'il faut contourner. Pour que les résidents sortent du "faire silence ", il s'agit de rechercher ce qui, dans le cadre spatio-temporel et relationnel, peut les inciter à "bouger » et à transformer le silence en prémices de la parole. Quels que soient les dispositifs, l'écoute apparait comme le vecteur de l'action des professionnels : être à l'écoute ${ }^{18}$ des présences, de leur humeur, d'une ambiance, qui bruissent plutôt qu'elles ne disent ${ }^{19}$. C'est la dimension affective, tonale, qui se donne à entendre et se prête à l'écoute. Écouter, n'estce pas se laisser imprégner ou pénétrer par le sonore qui peut être silence, jamais vraiment silencieux?

\section{BIBLIOGRAPHIE}

Archimbaud, Nicolas. 2013. Vie quotidienne et soins des personnes âgées dépendantes en milieu hospitalier. Une enquête filmique à l'hôpital Bretonneau (AP-HP, Paris 18ème). Thèse de doctorat. Nanterre : Université Paris-Ouest Nanterre (sciences de l'art - cinéma).

Augoyard, Jean-François. 2007-2008. La construction des atmosphères quotidiennes : l'ordinaire de la culture. Culture et recherche. $\mathrm{n}^{\circ}$ 114-115, p. 58-60.

Barus-Michel, Jacqueline (ed.). 1988. Représentation de l'espace chez les personnes âgées, le chez soi, l'espace-propre. Paris : Laboratoire de psychologie clinique, Paris VII. 
Binswanger, Ludwig. 1971 [1930]. Le rêve et l'existence. In : Introduction à l'analyse existentielle. Traduction Jacqueline Verdeaux \& Roland Kuhn, préface de Roland Kuhn et Henri Maldiney. Paris : Éd. de Minuit. p. 199-225.

Binswanger, Ludwig. 1998 [1932]. Le problème de l'espace en psychopathologie. Préface et traduction Caroline Gros-Azorin. Toulouse : Presses universitaires du Mirail.

Binswanger, Ludwig. 2002 [1957]. Le cas Suzanne Urban. Paris : Éd. Gérard Montfort.

Boltanski, Luc \& Thévenot, Laurent. 1991. De la justification. Les économies de la grandeur. Paris : Gallimard.

Bouisson, Jean. 2005. Psychologie du vieillissement et vie quotidienne. Marseille : Solal.

Bouisson, Jean. 2007. Vieillissement, vulnérabilité perçue et routinisation. Retraite et société. $\mathrm{n}^{\circ} 52$, p. 107-128.

Cabestan, Philippe. 2004. Préface. In : Chamond, Jeanine (ed.). Les directions de sens. Puteaux : Le Cercle Herméneutique. p. 9-17.

Caradec, Vincent. 2012 [3è édition]. Sociologie de la vieillesse et du vieillissement. Paris : Armand Colin.

Charbonneau, Georges. 2003a. Présentation. In: Granger, Bernard \& Charbonneau, Georges (eds.). Phénoménologie des sentiments corporels. I. Douleur, souffrance, dépression. Puteaux : Le Cercle Herméneutique. p. 17-23.

Charbonneau, Georges. 2003b. Les manifestations paradépressives. L'acédie entre fatigue et ennui. In : Granger, Bernard \& Charbonneau, Georges (eds.). Phénoménologie des sentiments corporels. II. Fatigue, lassitude, ennui. Puteaux : Le Cercle Herméneutique. p. 27-33.

Clément, Serge ; Mantovani, Jean \& Membrado, Monique. 1995. Vieillissement et Espaces urbains, modes de spatialisation et formes de déprise. Recherche financée par le PIRVilles-CNRS, CIEU, UTM et CJF INSERM 9406. Toulouse.

Dastur, Françoise. 2004. La phénoménologie en questions - Langage, altérité, temporalité, finitude. Paris : Librairie philosophique J. Vrin.

Eynard, Colette \& Salon, Didier. 2006. Architecture et Gérontologie. Peut-on habiter une maison de retraite? Paris : L'Harmattan.

Foucault, Michel. 1994. Des espaces autres. In : Foucault, Michel. Dits et écrits. IV, 1980-1988. Paris : Gallimard. p. 752-762.

Gaucher, Jacques. 2002. Ou la régression... quelle vieillesse ? Gérontologie et Société. n 101, p. 103-114.

Gaucher, Jacques. 1982a. Le temps rompu par l'âge. In : Guillaumin, Jean \& Reboul, Hélène (eds.). Le temps et la vie. Les dynamismes du vieillissement. Lyon: Chronique sociale. p. 47-52.

Gaucher, Jacques. 1982b. Les défauts de la mémoire comme signes de l'a-historicité de soi. In : Guillaumin, Jean \& Reboul, Hélène (eds.). Le temps et la vie. Les dynamismes du vieillissement. Lyon : Chronique sociale. p. $79-86$.

Goffman, Erwin. 1968. Asiles. Paris : Éd. de Minuit.

Goffman, Erwin. 1973. La folie dans la place. In : La mise en scène de la vie quotidienne. 2. Les relations en public. Paris : Éd. de Minuit. p. 313-361.

Gros, Caroline. 2009. Ludwig Binswanger. Chatou : Les éditions de la transparence. 
Guislain, Margot. 2012. EHPAD et Logements. AMC-Le Moniteur Architecture. $\mathrm{n}^{\circ}$ 219, p. 60-63. Jaujou, Nicolas ; Minnaërt, Éric \& Riot, Laurent. 2006. L'EHPAD : pour finir de vieillir - ethnologie comparée de la vie quotidienne en institution gériatrique. 2 tomes. Étude réalisée pour le Centre d'analyse stratégique par la Fondation Maison des Sciences de l'Homme.

Joseph, Isaac. 2001. Le reclus, le souci de soi et la folie de la place. In : Amourous, Charles \& Blanc, Alain (eds.). Erving Goffman et les institutions totales. Paris : L'Harmattan. p. 79-92.

Joseph, Isaac. 1996. Intermittence et réciprocité. In: Joseph, Isaac \& Proust, Joëlle (eds). La folie dans la place. Pathologies de l'interaction. Paris : Éd. de l'EHESS. p. 17-36.

Jullien, François. 2009. Les transformations silencieuses. Paris : Grasset.

Kaufmann, Jean-Claude. 2005. Le corps dans tous ses états : corps visible, corps sensible, corps secret. In : Bromberger, Christian ; Duret, Pascal ; Kaufmann, Jean-Claude et al. Un corps pour soi. Paris : PUF. p. 67-88.

Leroux, Martine. 2012. Les partages de l'ambiance en institution gériatrique. In : Thibaud, JeanPaul \& Siret, Daniel (eds.). Ambiances in action - Ambiances en actes : Proceedings of the 2nd international congress on ambiances - Actes du 2nd congrès international sur les ambiances, Montréal, 19 22 septembre 2012. Grenoble : Réseau international Ambiances : École nationale supérieure d'architecture de Grenoble. p. 695-700.

Leroux, Martine \& Bardyn, Jean-Luc. 2003. À l'écoute de l'hôpital. Grenoble : CRESSON : École nationale supérieure d'architecture de Grenoble. Contrat Assistance Publique / Hôpitaux de Paris.

Loffeier, Iris. 2011. Fabriquer du « bien-être ». Tension entre missions chez les personnels d'Établissements d'hébergement pour personnes âgées dépendantes (ÉHPAD) en France. Nouvelles pratiques sociales. vol. $24, \mathrm{n}^{\circ} 1$, p. $69-84$.

Maldiney, Henri. 1994 [1963]. Le dévoilement des concepts fondamentaux de la psychologie à travers la Daseinanalyse de L. Binswanger. In : Regard, parole, espace. Lausanne : Éd. L'âge d'homme. p. 87-101.

Maldiney, Henri. 2007 [1991]. De la transpassiblité. In : Penser l'homme et la folie. Grenoble : Éd Jérôme Million. p. 263-308.

Mallon, Isabelle. 2004. Vivre en maison de retraite. Rennes : Presses Universitaires de Rennes.

Marin, Claire. 2004. L'être et l'habitude dans la philosophie. Alter. nº 12, p. 149-172.

Marin, Claire. 2008. Hors de moi. Paris : Éd. Allia.

Minkowski, Eugène. 1932. Le problème du temps en psychopathologie. In : Recherches philosophiques 1932-33. Paris : Boivin et Cie. p. 231-256.

Minkowski, Eugène. 1968 [1933]. Le Temps vécu. Études phénoménologiques et psychopathologiques. Neuchatel : Delachaux et Niestlé.

Molinier, Pascale. 2009. Vulnérabilité et dépendance : de la maltraitance en régime de gestion hospitalière. In : Jouan, Marlène \& Laugier, Sandra (eds). Comment penser l'autonomie ? Entre compétences et dépendances. Paris : PUF. p. 433-458

Oury, Jean \& Dupassé, Marie. 2003. À quelle heure passe le train... Conversations sur la folie. Paris : Calmann-Lévy.

Ploton, Louis. 2001. La personne âgée - son accompagnement médical et psychologique et la question de la démence. Lyon : Chronique sociale. 
Ricœur, Paul. 2007. Vivant jusqu'à la mort ; suivi de Fragments. Paris : Seuil.

Richard, Jacques. 2004. Résilience et vulnérabilité. De l'ajustement des concepts en psychogérontologie. Gérontologie et société. n 109, p. 109-125.

Thibaud, Jean-Paul. 2010. La ville à l'épreuve des sens. In : Coutard, Olivier \& Lévy, Jean-Pierre (eds.). Écologies urbaines : états des savoirs et perspectives. Paris : Économica Anthropos. p. 198-213.

Thibaud, Jean-Paul. 2012. Petite archéologie de la notion d'ambiance. Communications. $n^{\circ} 90$, p. 155-174.

Thomas, Hélène 2008. Vulnérabilité, fragilité, précarité, résilience, etc. Esquisses. ${ }^{\circ} 13$. Disponible sur : http://www.reseau-terra.eu/article697.html (consulté le 12 février 2016).

Worms, Frédéric. 2006. Les deux concepts du soin. Vie, médecine, relations morales. Esprit. 1, p. 141-156.

\section{NOTES}

1. Hélène Thomas s'intéresse au passage de ces notions courantes dans les sciences sociales et en médecine. «Le terme de vulnérabilité [vulnerability] a percé, conjointement dans la littérature psychiatrique, psychologique, pédiatrique et psychanalytique d'une part, et gériatrique d'autre part, sous son synonyme de fragilité [frailty, fragility, fragilization] au début des années 1970. Il a commencé à se diffuser à partir des années 1980 dans les articles en anglais avec ses corrélats de résilience [resiliency] et [invicibility] puis s'est répandu massivement dans les années 1990, dans une traduction en français proche du sens originel » (Thomas, 2008, p. 4).

2. Enquête sous forme d'observations et d'entretiens, d'une part dans les services gériatriques de deux hôpitaux parisiens [Leroux \& Bardyn, 2003], et d'autre part dans un EHPAD à Paris en 2008. Par ailleurs, l'accompagnement de personnes âgées en institution en tant que bénévole d'une association nous a familiarisée aux ambiances des espaces collectifs.

3. Binswanger a développé sa pensée à partir de la phénoménologie de Husserl et de Heidegger. Il a introduit la Daseinanalyse, ou analyse existentielle, qui désigne une nouvelle méthode de compréhension de l'être malade fondée sur la "proximité » - plutôt que sur l'intersubjectivté de la présence malade (mode déficient de la présence) et du psychiatre (mode authentique du comprendre), et cette proximité «n'est possible que par ce que Ludwig Binswanger appelle la structure de la Présence commune » (Maldiney, 1994, p. 90-91).

4. Voir à cet égard la préface de Caroline Gros à la conférence de Binswanger Le problème de l'espace en psychopathologie (1998 [1932]), ainsi que la partie consacrée à l'espace dans son ouvrage Ludwig Binswanger (Gros, 2009) ; voir également l'analyse de Jean-Paul Thibaud qui, faisant une "Petite archéologie de la notion d'ambiance", écrit: "Pour comprendre la façon dont la psychopathologie a thématisé la notion d'ambiance, il faut sans doute revenir aux distinctions opérées entre diverses formes d'espace » (Thibaud, 2012, p. 161).

5. Nous retenons ici cette expression plutôt que "direction significative " adoptée par les traducteurs (Jacqueline Verdeaux \& Roland Kuhn) de L'introduction à l'analyse existentielle (Binswanger, 1971). Direction de sens ne dissocie pas la direction spatiale et la signification ; « il y a un seul mouvement de l'existence dont les directions de sens sont aussi bien spatiales que linguistiques » (Cabestan, 2004, p. 11).

6. C'est dans le discours des malades qui ne font que recourir au langage commun et à sa force évocatrice que Binswanger va repérer les directions de sens qui, au-delà de la métaphore, expriment le mouvement de l'existence ; ainsi l'expression «tomber des nues » n'indique-t-elle pas la chute (Binswanger, 1971, p. 200) de la présence? 
7. La juxtaposition serait la caractéristique de la temporalité des schizophrènes, la pénétration celle des maniaco-dépressifs. Mais cette opposition ne s'avérera pas si pertinente pour distinguer ces deux pathologies.

8. «Le caractère essentiel des institutions totalitaires est qu'elles appliquent à l'homme un traitement collectif conforme à un système d'organisation bureaucratique qui prend en charge tous ses besoins, quelles que soient en l'occurrence la nécessité ou l'efficacité de ce système » (Goffman, 1968, p. 48) ; "L'institution totalitaire est un mixte social, à la fois communauté résidentielle et organisation réglementée » (p. 54).

9. Voir Leroux, 2012, qui aborde la question du «fond commun du sensible» en institution gériatrique.

10. Lieu radicalement autre dans lequel "on place les individus en état de crise", "dont le comportement est déviant »; qui a « le pouvoir de juxtaposer plusieurs espaces » (Foucault, 1984, p. 757-758).

11. "Sur la ZAC Claude Bernard, un immeuble superpose des logements en accession à un établissement pour personnes âgées dépendantes »; le hall d'entrée est intergénérationnel (Guislain, 2012, p. 60).

12. L'espace propre « est circonscrit par une enveloppe qui entoure l'espace occupé par le corps propre du sujet. La maîtrise psycho-motrice (liée à l'existence de handicaps éventuels, à l'état de santé), le schéma corporel induisent l'organisation de cet espace » (Barus-Michel, 1988, p. 61).

13. «Plus qu'une place, un lieu, le rôle serait une sorte d'inscription et de reconnaissance de l'individu dans sa constellation sociale. Il s'agirait d'un ensemble de possibilités offertes par le groupe à un être humain pour réaliser et réguler sa manière d'être au monde » (Gaucher, 1982b, p. 82).

14. «"Aller vers la tombe", "aller vers les cieux", [...] ces expressions ne sont pas de simples métaphores dans notre esprit. C'est que vraiment l'imprégnation de notre être par des facteurs matériels est ressentie comme un poids qui nous attire vers la terre, vers en-bas, tandis qu'au contraire, d'autre part, nous nous sentons comme emportés par notre élan vers le haut » (Minkowski, 1968, p. 131).

15. " "Passible" signifie "capable de pâtir, de subir" et cette capacité implique une activité, immanente à l'épreuve, au pathein, qui consiste à ouvrir son champ propre de réceptivité " (Maldiney, 2007, p. 265). La transpassibilité, est ouverture à l'événement, "ouverture sans dessein ».

16. À propos de Folie dans la place (1973): «Lorsque Goffman dit de la folie qu'elle est une "inconvenance situationnelle", il faut donc non seulement entendre qu'elle porte atteinte à une strate de sens plus profonde que celle des normes de civilité (le fou est plus qu'un déviant), mais souligne que c'est le cadre de l'expérience sociale élémentaire, en tant qu'elle suppose une intelligence partagée, qui est troublé » (Joseph, 1996, p. 19).

17. «Le care, concept intraduisible en français, désigne le souci des autres - plus largement du vivant - réalisé à travers des activités concrètes, un travail (caring). Le domaine du care excède donc largement celui du soin aux personnes malades " (Molinier, 2009, p. 433).

18. Nous nous référons ici au livre de Jean-Luc Nancy, À l'écoute (2002), dont le propos tourne autour d'une « résonance fondamentale »: «Peut-être faut-il que le sens ne se contente pas de faire sens (ou d'être logos), mais en outre résonne » (p. 19).

19. S'intéressant au registre auditif, Jean-Luc Nancy remarque : "Entendre" veut dire aussi "comprendre", comme si "entendre" était avant tout "entendre dire" (plutôt qu'"entendre bruire")... » (Ibid., p. 18). 


\section{RÉSUMÉS}

Cet article s'intéresse au vécu de la vulnérabilité chez les personnes âgées en institution gériatrique. Les expressions « rester sans voix » et «faire silence » rendent compte, du point de vue phénoménologique, de l'épreuve que constitue la découverte de soi comme être vulnérable, parce que vieux, et de l'installation du sentiment de vulnérabilité. Le recours à la notion d'ambiance permet d'envisager la tonalité des "présences " - traduction du terme Dasein que préconise Binswanger - et d'interroger les facteurs d'ambiance dans les trois dimensions qui se conjuguent dans le silence vécu: spatialité, temporalité et altérité. Ces facteurs propres à l'institution sont-ils susceptibles de «mettre au silence » les résidents?

This article looks at the experience of vulnerability among elderly people in geriatric institutions. In the point of view of the phenomenological perspective, the terms "remain speechless " and "stay in silence " express the hardship of discovery of the self as a vulnerable being, because of old age, and the instilling sense of vulnerability. The use of the notion of ambiance allows for the consideration of the tone of «presence " - translation of Dasein that Binswanger proposed - and to question atmospheric factors in all three dimensions that we find combined in the lived silence: spatiality, temporality and otherness. Are these specific factors of the institution capable of « putting to silence » the residents?

\section{INDEX}

Mots-clés : silence, vulnérabilité, présence, tonalité, ambiance, institution gériatrique Keywords : silence, vulnerability, presence, tone, ambiance, geriatric institution

\section{AUTEUR}

\section{MARTINE LEROUX}

Chercheuse associée depuis 1987 au CRESSON UMR 1563, ENSAG, Grenoble, France martine.leroux@grenoble.archi.fr 